\title{
Infrared Thermography study of the local heat transfer coefficient in front of a single finned tube
}

\author{
By M. Sanhaji*, D. Bougeard*, A. El abbadi*, \\ S. Russeil*, M. Nacer bey*, B. Baudoin* \\ *Ecole des Mines de Douai, Département Energétique Industrielle, DOUAl, France
}

\begin{abstract}
The objective of this study is to investigate the influence of the inlet flow velocity on local heat transfer mechanisms in a single finned tube assembly. Within this framework, an Infrared Thermography technique was used to determine the convection heat transfer coefficient distribution in front of the tube, then results were confronted to the flow structure obtained using a PIV system by M. Nacer-Bey [1]. Flow velocity is found to have a considerable effect; as it increased, horseshoe vortices size and intensity increased causing thermal peaks augmentation. We distinguish a separating zone whose size is depending on Reynolds number and characterised by a high heat transfer. Also, the position of both horseshoe vortices and their associated thermal peaks was studied. Results are analysed and correlations between heat and momentum transfer are highlighted.
\end{abstract}

\section{Introduction}

Heat exchangers have many application areas like heating, air conditioning, refrigeration, engine cooling and many other applications in the field of thermal engineering. Because of this wide use, studying heat transfer and flow structure inside heat exchangers is important as means of improving their performances.

Basic plate-fin-and-tube heat exchangers geometry corresponds to a circular cylinder bounded by two fins. At each fin-cylinder junction, the flow is governed by the combined effect of the fin boundary layer and the stagnation flow upstream the cylinder. This involves the formation of the well-known horseshoe vortex (HV) structure around the tube causing high heat transfer rates in a U-shaped area.

Relating to this, several studies were focused on the flow structure and heat transfer characteristics of finned tubes $[2,3]$. Some, which are in relation to our study, treated more particully the inlet flow velocity effect on the heat transfer in front of the tube:

Schüz and Koottke [4] used a mass transfer method, based on a color reaction to investigate the effect of varying the basic geometric design parameters on the flow field and the heat transfer. They showed that some geometric parameters play a crucial role on the heat transfer enhancement due to the HV structure. But their study was mainly qualitative and no quantitative comparison was made with flow structure investigation.

Onishi et al. [5] developed an unsteady numerical code to study the flow topology of a single row heat exchanger. Their study concerned middle Reynolds number range. It confirmed the influence of flow velocity on heat transfer around the tube junction; They show that as Reynolds number increased, heat transfer enhancement caused by horseshoe vortex is intensively generated near the tube base. 
Kim et al. [6] used the naphthalene sublimation technique to examine the influence of fin spacing and Reynolds number on flow and heat transfer in a plate fins/circular tube assembly. They show that the horseshoe vortex, formed in front of the tube, gives significant increases in total heat transfer rate. Moreover, they note the apparition of a smaller subsidiary thermal peak when Reynolds number is increased and suggest that it is caused by a secondary vortex formation. However, although their study was focused on local flow and heat transfer, Kim et al. do not paid a major attention to the relation between horseshoe vortices intensity and that of their associated thermal peaks.

Thus, though many authors were interested in how flow velocity variations affect the global heat transfer performance of heat exchangers, detailed investigations on this topic are still required in order to describe the impact of this parameter on horseshoe vortices structure and so, on the local heat transfer.

Recently, M. Nacer-Bey et al.[1], studied in detail the influence of flow velocity on the horseshoe vortex structure formed at the tube-fin juncture using a Particle Image Velocimetry (PIV) system. They demonstrated that parameters like horseshoe vortices number, size and intensity increased with the increase of the flow velocity.

In this paper, the PIV experimental test bench is not presented. Only the Infrared set-up is described. We discuss how the inlet flow velocity impact the convection heat transfer coefficient over a single finned tube trough a confrontation between our thermal results (obtained by Infrared thermography) and M.N Bey results (obtained using a PIV system). During the experiment, the fin spacing $E / D$ was fixed equal to 0.2 and measurements were performed for Reynolds numbers (based on tube diameter) between 1400 and 3840.

\section{Infrared thermography investigation}

\subsection{Experimental bench description}

The infrared set-up consists in a small wind tunnel with a single finned tube test model as shown in figure 1. It can be divided into three sections:

- The first section is an air conditioning volume. The air is cooled until $14^{\circ} \mathrm{C}$ and then ducted to the test section through a honeycomb in order to reduce the turbulence intensity. In the exit of the isothermal room, an inlet contraction which had a 20:1 area reduction, was designed using Morel [7] correlation in order to give a roughly uniform velocity profile at the inlet of the test section.

- $\quad$ The test section is $450 \mathrm{~mm}$ width and its height can vary from 15 to 25 $\mathrm{mm}$ in order to test different fin spacing. An infrared camera is placed on the upper side of the test section allowing the measurement of fin temperature field through an IR transparent window (a ZnSe porthole which is essentially transparent in the range of $(8-12 \mu \mathrm{m})$ sensitivity wave length). A removable IR emitter is used to heat the fin surface. It leaves the place to the camera once the initial temperature of the fin is reached (see chapter 3.1).

- In the downstream circuit, a system of two flow control valves and a derivation pipe allow the pumping of air either inside or outside the isothermal room. The air is finally ducted to a flowmeter before being discharged into the atmosphere.

The investigated zone size (figure 2 ) is $136 \times 170$ pixel $^{2}$. It corresponds to a surface of $108 \times 135 \mathrm{~mm}^{2}$. Further description of the test facilities can be found in Bougeard [8]. 


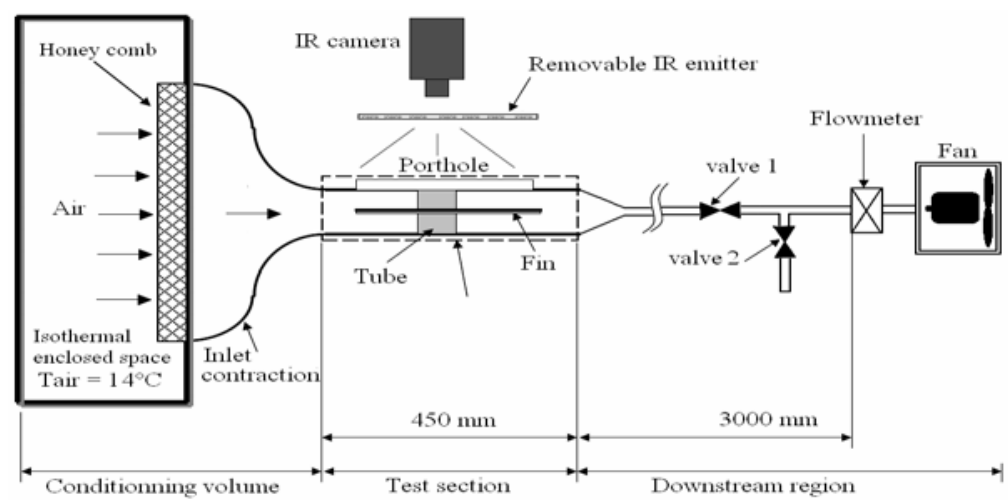

Fig.1. Experimental test bench

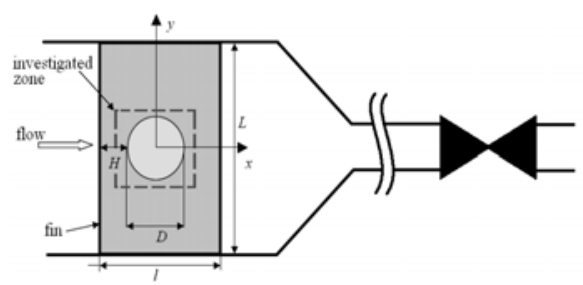

Fig.2. Top view of the test section

\subsection{Test mode}

The plate-finned tube model used consists of a single tube fixed to a fin as shown in figure 3 . The study is performed on a scaled up model whose dimensions have been chosen in order to insure an optimal spatial resolution for the IR Thermography investigation. Dimensionless parameters corresponding to the different cases studied are summarised in Table 1. E/D is chosen in similarity with usual compact exchangers values used in automotive industry. The distance, $\mathrm{H}$, between the tube position and the fin leading edge is chosen in order to support horseshoe vortices formation as shown in Kim et al. study [6]. Different Reynolds numbers tested are shown in table 2. The coordinate system origin is placed at the tube center as shown in figure 3.
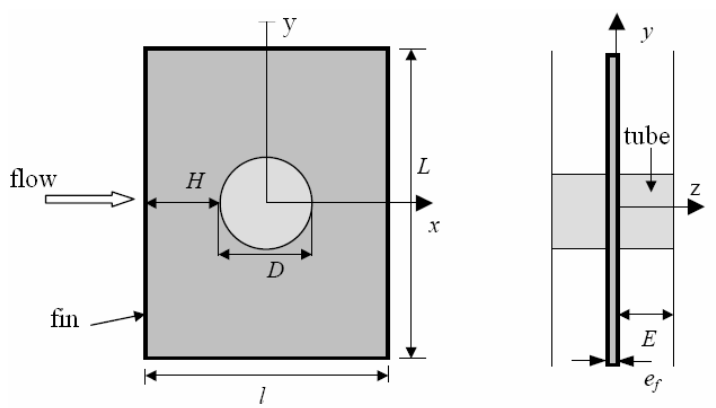

Fig.3. Geometry 


\begin{tabular}{|c|c|}
\hline$E / D$ & 0.2 \\
\hline$L / D$ & 7.09 \\
\hline$I / D$ & 3.7 \\
\hline$H / D$ & 1.357 \\
\hline$e_{f} / D$ & 0.0086 \\
\hline
\end{tabular}

Table 1. Test model dimensions

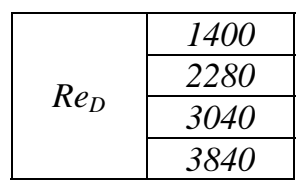

Table 2. Reynolds numbers tested

\subsection{Temperature measurements by infrared thermography}

The temperature field is measured using an infrared camera (AGEMA 900 Long Wave) equipped with $\mathrm{HgCdTe}$ sensor which is sensitive to 8-12 $\mu \mathrm{m}$ wavelength and cooled by a Stirling engine. The infrared camera is placed at its minimal focalisation distance in order to give maximal spatial resolution. Thermal images are captured at a frequency of 15 frames per second (15 hertz).

In our study, the fin is made of PVC and painted with a high emissivity coating $(\varepsilon \approx 0.95)$. The analog signal get by the infrared camera is digitally recorded with a 12-bit dynamic range using a specific high accuracy signal-conditioning unit (ADDELIE $®)$. To convert this signal (expressed by numeric level (NL)) into temperature, an in-situ calibration technique is used. A special high conductivity plate coated with the same black painting as the fin and placed instead of it. This calibration plate can be considered very closed to an ideal blackbody with very low reflection. In order to enhance the thermal resolution, the calibration curve uses the whole dynamic range (12 bit) of the model temperature variation during the experiment as can be seen in figure 5 .

One major limitation of infrared systems is thermal resolution. Indeed, some degradations are introduced by the noise detector itself and by analog-to-digital (A/D) converter when sampling the analog signal provided by the detector. To deal with this problem, the initial temperature, $T_{i}\left(x, y, t_{i}\right)$ and the final one $T_{f}\left(x, y, t_{f}\right)$, are time averaged over one second period. Thus the random noise of temperature measurement is reduced and the sensivity of the camera is enhanced. Figure 4 shows the noise standard variation according to the number of images. As we can see the noise is well reduced (less than 2 numerical level).

Another major limitation of infrared systems is the spatial resolution. Degradations that affect spatial resolution concerns high spatial frequencies signals existing on the thermal scene. Their amplitude is lowered by the infrared scanner as a consequence of the spatial averaging effect over the IPA (Instantaneous Projected Area). In order to limit this phenomenon, we placed the camera at its minimal focalisation distance. Moreover, to enhance spatial resolution, we use a specific digital image restoration technique (deconvolution technique) by mean of a two dimensional Wiener filter. Details on this technique can be found in Bougeard et al. [9]. Thanks to these treatments, our experimental technique allows accurate measurements of the fin temperature with spatial variation up to three pixels width with little smoothing. 


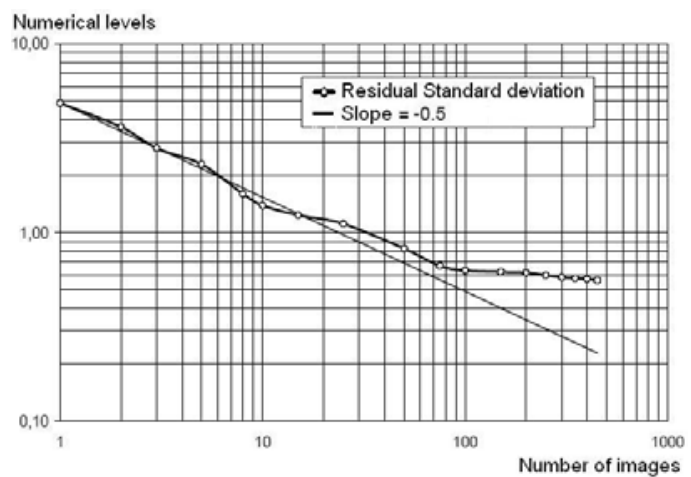

Fig.4. Noise standard variation according to the number of averaged images

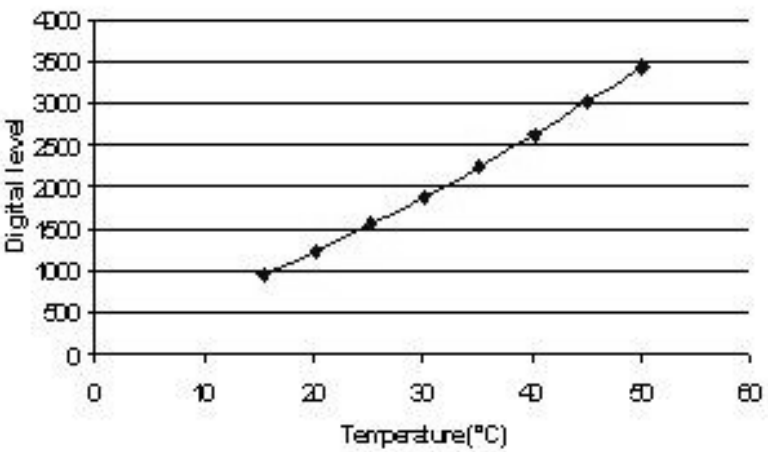

Fig.5. Calibration curve

\section{Experimental method}

\subsection{Experimental procedure}

Initially, the test model is warmed up until $40^{\circ} \mathrm{C}$, using a removable infrared emitter, before being cooled by convection. First, the blower is on and the control valve (1) is closed while control valve (2) is opened implying no air-flow inside the test section. During this period, the infrared emitter heats up the fin until $40^{\circ} \mathrm{C}$. Then the infrared emitter is manually removed, and control valve (1) is opened while control valve (2) is closed steadying air flow in the test section. During this cooling period, the infrared set up is used to record the temperature drop between initial instant $t_{i}$ and final instant $t_{f}$. The infrared film acquired is treated after with specific program written using Matlab code, and the heat transfer coefficient is then determined using Eq. (3)

\subsection{Heat transfer coefficient calculation}

The experimental method approached the lumped Capacitance Method (LCM) used by Kim et al. [10] and Tourreuil [11]. In those authors previous work, the experimental process consists in suddenly warming up an experimental model. The 
local convection coefficient is then deduced from the local time variation of model temperature. In our case, the fin is divided to many small elements. The heat fluxes balance in an element gives:

$$
\rho_{\text {fin }} C p_{\text {fin }} e_{\text {fin }} \frac{\partial T_{\text {fin }}}{\partial t}=\Phi_{\text {conv }}+\Phi_{\text {cond }}+\Phi_{\text {rad }}
$$

If we made the three following assumptions:

1- The temperature is constant across the plate thickness (i.e., negligible temperature gradient in the $\mathrm{z}$ direction),

2- Conductive and radiative fluxes are negligible,

3- The heat transfer coefficient is considered as constant during the experiment, and taking into account that the heat transfer coefficient is equal on each side of the fin, the preceding equation gives :

$$
\rho_{\text {fin }} C p_{\text {fin }} e_{f i n} d x d y \frac{\partial T_{\text {fin }}}{\partial t}=2 h(x, y) d x d y\left(T_{\text {air }}-T_{f i n}\right)
$$

Time integration of this equation in the time gives:

$$
h(x, y)=\frac{\rho_{\text {fin }} C_{p} e_{f i n}}{2 \Delta t} \ln \frac{T_{\text {air }}-T_{f i n}\left(x, y, t_{i}\right)}{T_{\text {air }}-T_{f i n}\left(x, y, t_{f}\right)}
$$

This method is known as LCM method. It is verified only if the three assumptions are validated.

The first assumption is validated thanks to an appropriate choice of fin properties. The thickness and material of the fin (PVC fin, Thickness $0.5 \mathrm{~mm}$ ) were selected in order to give a low Biot number value. In fact Kim [10] has shown the reliability of the method when the Biot number is less than 0.058. In our case the mean Biot number of the fin is equal to 0.046 .

The second assumption is validated numerically by El abbadi [12]. The energy balance equation is resolved for a PVC fin (taking into account the conduction). The result shows that the surface averaged heat transfer coefficient obtained differs only of $2 \%$ comparing with the case when the conduction flux is neglected. Anyway, one should keep in mind that locally high spatial variation of the Heat Transfer Coefficient (HTC) can induce a rather important increase of conductive flux.

In order to minimise the radiative flux influence on our measures, the fin temperature averaged between the beginning and the end of the experiment is taken very close to that of the environment. Hence the air temperature at the inlet of our test model is taken weak enough $\left(15^{\circ} \mathrm{C}\right)$, and the radiative flux influence is considered to be minor. The validation of this assumption can be found in El abbadi [12].

The third assumption supposes that $h$ is constant during the cooling period. This assumption is used by several authors. In our case, we choose a sufficiently short period of cooling so that the variation of the fin temperature during the experiment be relatively weak when compared to the difference between air and fin temperatures; i.e. $\left(T_{f}-T_{i}\right)_{\text {fin }}<<\left(\left(T_{\text {mean }}\right)_{\text {fin }}-\left(T_{\text {mean }}\right)_{\text {air }}\right)$. Nevertheless, it is necessary that the fin temperature variation be sufficient to limit uncertainties of measurement. Indeed, experimental uncertainty on the determination of convection coefficient is strongly dependent on time variation of temperature $\left(T_{f}-T_{i}\right)$ fin. If this last is very low the noise 
of measurement, inherent to the thermography device, involves too much significant errors of measurement.

The relative uncertainty of the heat transfer coefficient (HTC) averaged over all the surface is $\pm 7 \%$.

For more details about the mathematical calculation and uncertainties of the HTC refer to El abbadi [12].

\section{Results and discussion}

\subsection{Problem statement-Fluid mechanics}

Figure 6 represents an explanatory diagram showing the flow topology generally obtained in front of a single finned tube. M.Bey notes the presence of one vortex, two vortices or three vortices according to the flow velocity (figure 8). He distinguishes a separating zone which delimits all the horseshoe vortex system in front of the finned tube. This zone widens with Reynolds increase because of the formation of new vortices ahead of the main one and also because of the augmentation of their size. Another important observation deferred by M. Nacer-Bey is the presence of a downwash region, induced by main vortices (N1, N2) and located where the flow is directed towards the wall.

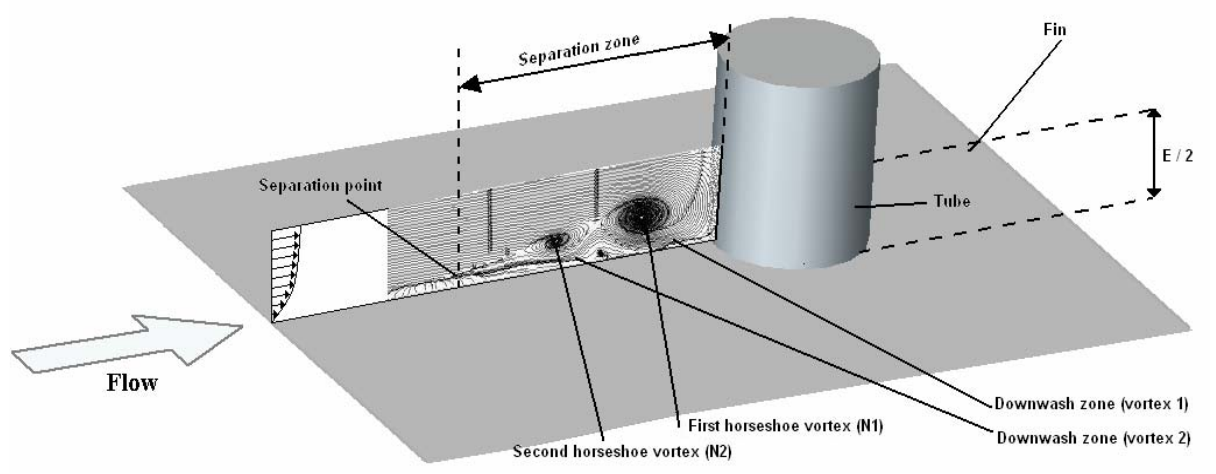

Fig.6. Explanatory diagram: flow topology in front of a single finned tube.

\subsection{HTC distribution on the fin}

Cartographies of the local heat transfer coefficient (HTC) are presented in figure 7 . We note that the heat transfer coefficient is strongly influenced by the inlet flow velocity. U-shaped thermal imprints appear progressively around the tube and their sizes are increasing with the velocity increase. That can be explained thanks to flow visualisations established by M. Nacer-Bey [1]. Observations figure 8 showed that for each thermal imprint, a corresponding horseshoe vortex is visible. Moreover, the area influenced by horseshoe vortices is more significant in the case of high speeds involving the increase of thermal transfer there. Indeed, heat transfer enhancement started from the separating point and spreads out over the entire separation zone. 


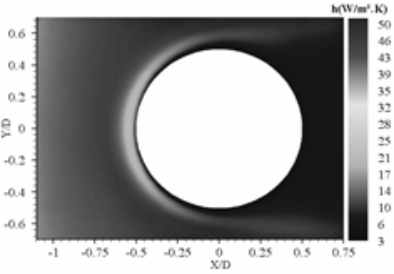

(a) $R e_{D}=1400$

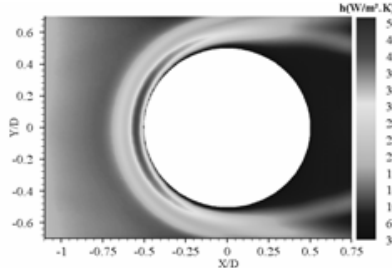

(b) $R e_{D}=3040$

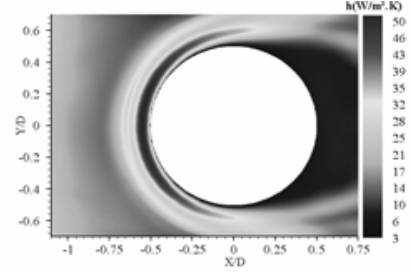

(C) $R e_{D}=3840$

Fig.7. Cartographies of the local HTC for different ReD number, $E / D=0.2$

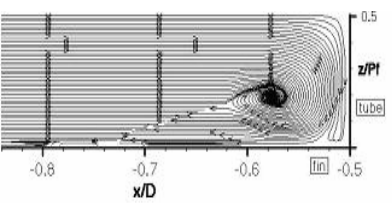

(a) $R e_{D}=1400$

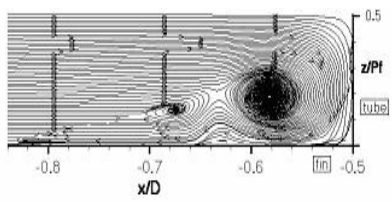

(b) $R e_{D}=3040$

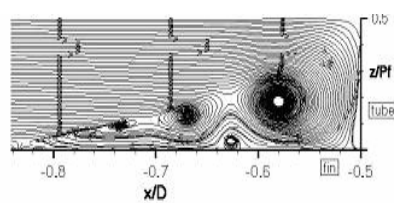

(C) $R e_{D}=3840$

Fig.8. Flow structure in front of a single finned tube for different $R_{D}$ number, $E / D=0.2$

For better understanding, Figure 9 gives an illustration of the HTC variation through the tube center line (the line located on the fin surface upstream the tube, $y / D=0)$. Thermal peaks due to vortices horseshoe (N1 and N2 as depicted in figure 6 ) appear progressively with velocity increase. In particular, the second one, caused by the presence of a subsidiary horseshoe vortex (N2), and which becomes visible from $\mathrm{Re}=2280$. We can also notice that thermal peaks intensity is depending on vortices size and vorticity. This is clearly visible when comparing the thermal peak nearest the tube (figure 9) and the first vortex (N1) vorticity (figure 10). Both of them are increasing with Reynolds number increase.

In figure 11, the x-positions of horseshoe vortices (N1, N2) and their corresponding thermal peaks upstream the tube are illustrated. We can see that both of them are not moving when varying the Reynolds number. On the other hand, even if their positions are close, that of the thermal peak doesn't exactly correspond to that of the vortex center. It seems to be located in the downwash region nearer the tube base (figure 6). Indeed, in this region, the boundary layer is thinned by the strong downflow allowing the enhancement of the heat transfer there.

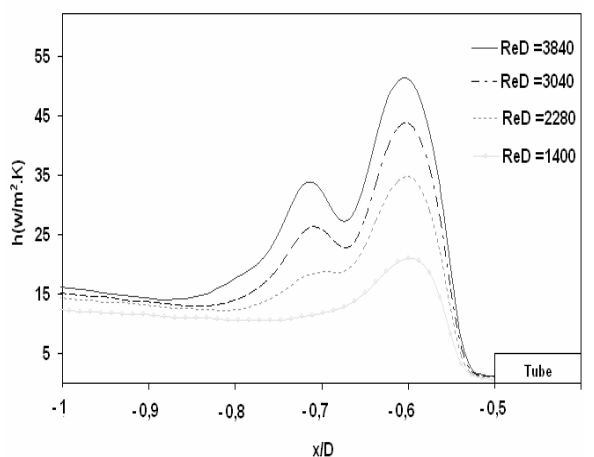

Fig.9. HTC variation along the tube center line

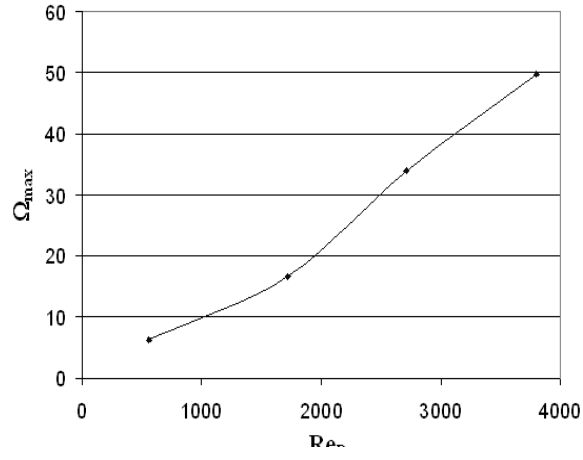

Fig.10. Influence of flow velocity on first vortex vorticity 


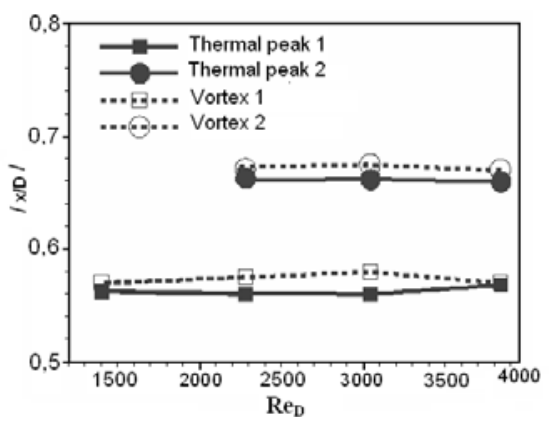

Fig.11. Horseshoe vortex and thermal peak positions

\section{Conclusion}

Current work aims at studying the effect of the flow velocity on the heat exchange by linking fluid mechanics and heat transfer. It is found that the flow structure can be related to HTC variations in front of the tube. A strong dependence of Reynolds number on the convection heat transfer is also observed as the flow topology pointed it out. Most important conclusions can be summarised as follows:

- The heat transfer enhancement is spread out over the entire separation zone which delimits the horseshoe vortex system in front of the finned tube.

- As Reynolds number increased, horseshoe vortices size and vorticity increased causing thermal peaks augmentation.

- When the flow velocity rises, the position of horseshoe vortex slightly differs from that of its corresponding thermal peak which is situated in the downwash region.

\section{NOMENCLATURE}

Bi Biot number, $h e_{f} / 2 \lambda_{\text {fin }}$

Cp Specific heat of the fin $[\mathrm{J} / \mathrm{kgK}]$

$D \quad$ Tube diameter $[\mathrm{m}]$

e Thickness [m]

$E \quad$ Fin spacing [m]

$h \quad$ Heat transfer coefficient $\left[\mathrm{W} / \mathrm{m}^{2} . \mathrm{K}\right]$

$H \quad$ Distance between tube position and the fin leading edge [m]

I $\quad$ Fin height [m]

$L \quad$ Fin length [m]

$T$ Temperature $[\mathrm{K}]$

$R e_{D} \quad$ Reynolds number, $D U_{0} / v$

$U_{0} \quad$ Inlet flow velocity [m/s]

$\rho$ density $\left[\mathrm{kg} / \mathrm{m}^{3}\right]$

$\Omega_{\max }$ Maximum measured vorticity at the first vortex $\left[\mathrm{m}^{2} / \mathrm{s}\right]$

$\lambda \quad$ Thermal conductivity [W/m.K]

$\phi \quad$ Heat flux $\left[\mathrm{W} / \mathrm{m}^{2}\right]$

\section{SUBSCRIPTS}

fin Relative to fin

i initial

f final

cond Conductive

conv Convective

rad Radiative

in Inlet

air Air

mean Time averaged 


\section{REFERENCES}

[1] M. Nacer-Bey, S. Russeil, B. Baudoin, PIV study of steady horseshoe vortex system in front of a single plate-finned tube: effect of fin spacing and velocity, Experimental Thermal and Fluid Science, submitted.

[2] F.E.M. Saboya, E.M. Sparrow, Local and average transfer coefficients for one-row plate fin and tube heat exchanger configurations, ASME J. Heat Transfer 96(1974) 265-272.

[3] F.E.M. Saboya, E.M. Sparrow, Transfer characteristics of two-row plate fin and tube heat exchanger configurations, Int. J. Heat Mass transfer 19 (1976) 41-49.

[4] G. Schüz and V. Koottke, Visualisation of flow, heat and mass transfer on finned tubes in cross flow, $4^{\text {th }}$ Int. Symposium on Flow Visualisation, Paris (1987) 637-642.

[5] H. Onishi, K. Inaoka, K. Suzuki, A three-dimensional unsteady numerical analysis for a plate-finned tube heat exchanger in the middle Reynolds number range, Proceedings of the Third Int. Conference on Compact Heat Exchangers and Enhancement Technology for the Process Industries (2001) 9-16.

[6] J.Y. Kim and T.H. Song, Microscopic phenomena and macroscopic evaluation of heat transfer from plate fins/circular tube assembly using naphthalene sublimation technique, Int. J. Heat Mass Transfer 45 (2002) 3397-3404.

[7] T. Morel, Design of 2-dimensional wind tunnel contractions, Journal of Fluids Engineering, June (1977) 371-378

[8] D. Bougeard, Infrared thermography investigation of local heat transfer in plate fin and two-tube rows assembly, Int. J. Heat and Fluid flow, submitted.

[9] D. Bougeard, J-P. Vermeulen, B. Baudoin, Spatial resolution enhancement of an IR system by image restoration techniques, In Proceedings EUROTHERM seminar 42, Quantitative Infrared Thermography QIRT 94, pp.3-8, Sorrento, Italy, 23-26 August 1994.

[10] Y.Y. Kim, K. S. Kim, T., G. H. Jeong, S. Jeong, An experimental study on the quantitative interpretation of local convective heat transfer for a plate fin and tube heat exchanger using the lumped capacitance method, Int. J. Heat Mass Transfer 49 (2006) 230-239.

[11] J. Tourreuil, Etude des performances thermiques d'un échangeur de chaleur compact muni de promoteur de tourbillons - développement de techniques de mesures locales, Thèse de doctorat, Université de Valenciennes et du Hainaut Cambrésis, Ecole des mines de Douai, 2002.

[12] A. El abbadi, Développement de méthodes de mesure des transferts convectifs par thermographie infrarouge: Applications aux ailettes d'échangeurs à hautes performances, Thèse de Doctorat, Université de valenciennes et du Hainaut Cambrésis, Ecole des mines de Douai, 2006. 